\title{
Veterinarian and Mental Health
}

\section{Amaan Ghazi*}

B.V.sc and AH, Apollo College of Veterinary Medicine, India

*Corresponding Author: Amaan Ghazi, B.V.sc and AH, Apollo College of Veterinary

Medicine, India.
Received: August 12, 2021

Published: August 23, 2021

(C) All rights are reserved by Amaan Ghazi.

\section{Abstract}

Approximately one million people attempt and die by suicide each year due to their helplessness towards their psychological well-being.

Numerous researchers and studies have claimed that Veterinarians too suffer from deteriorating mental health while contributing their share of duty and responsibility to the society.

Our psychological health generally goes ignored but that of veterinarians isn't considered for "they are not dealing with humans" but definitely living beings. Despite many researches that have conveyed shocking results about the same, "it is discussed or considered lesser".

In this paper such researches and studies have been reviewed and discussed while providing more suggestions to add onto in order to improve psychological well-being of veterinarians and become aware about the same.

Keywords: Veterinarian; Mental Health; Psychological Issues; Depression; Anxiety; Sleeplessness; Euthanasia; Suicidal Ideation

\section{Abbreviations}

RR: Relative Risk; WHO: World Health Organization; VCR: Veterinary Client Relationship; VMAE: Veterinary Medical Association Executives; SCAVMA: Student Chapter of the American Veterinary Medical Association

\section{Introduction}

\section{Mental health}

According to WHO, "Mental Health is a state of well-being in which an individual realizes his or her own abilities, can cope with the normal stresses of life, can work productively, and is able to make a contribution to his or her community" [1].

Mental Health at work place has not been a new news, it has existed in the place from more than an epoch. In this century, Veterinary Professionals and students are more experiencing work- related anxiety, depression, sleep-deprivation, stress etc. Later which comes out as suicidal ideation, which is thoughts related to suicide and at last suicide. Other than any profession, Veterinary have highest rate of mental health issues, and suicides.

\section{Mental illness}

Psychological Illness such as depression, bipolar, anxiety-disorder, insomnia, suicidal ideation, loss of compassion, burnout etc. arises gradually due to regular exposure to Euthanasia (especially in companion animals), necropsy, workload, poor veterinary client relationship (VCR) and intensive surgery. Later results in substance abuse, suicidal ideation and suicide.

As new-emerging disease are rising, and the culture of keeping different species as pets, are overburdening the veterinarian and veterinary students is also the reason for mental health illness development. 


\section{Methods}

- $\quad$ Articles, research papers, survey data are collected and reviewed from many journals on Mental Health of Veterinarian

- $\quad$ Study and representation of statistical survey data done by different agencies.

- This article revolves around the issues and what could be the cause for these psychological issues.

\section{Results and Discussion}

Agencies such as SCAVMA, American Society of Veterinary Medical Association Executives (VMAE), Alabama State Veterinary Association have conducted anonymous survey, which had questionnaire related to veterinary medicine, and mental health. In UK, a study on Relative Risk was conducted, in which the rates of suicide were compared between the general population, and other health professionals.

SCAVMA Survey concluded, that $88 \%$ found veterinary medicine stressful, $19 \%$ indicated that they have been diagnosed with a mental illness, $13 \%$ had tried to consider and attempted suicide.

Alabama State Survey, invited 1455 licensed Veterinarians, out of which 701 attempted the survey, 57\% men and 43\% women vet completed the questionnaire, $66 \%$ of the participants have stated they have been diagnosed with mental illness, $24 \%$ had considered committing suicide form the beginning of the vet school, and the rest had committed suicide.

Directive Survey also showed the same results, 96\% believed increases stress has been placed on vets over past 10 years, 95 found burnout is a serious issue, while other had been diagnosed with mental illness, or had a suicidal ideation and tried committing suicide [2].

United Kingdom's Relative Risk Study showed a clearer picture of mental illness and led issues from it. It cited the difference of suicidal prevalence in general population, veterinary and other health-care professionals. Among them, Veterinarians had a highest suicidal prevalence rate of suicide. Male veterinarians between the age of 45 to 65 , had a relative risk of 5.62 times more than that of general population and other health-care professionals. Female veterinarians have highest RR of 7.2 times that of non-vet professionals, they have a RR of 4.5 [3].

On the other hand, studies on sleep quality and sleeplessness were conducted by the accordance of Virginia Tech Institutional Review Board, which evaluated the Pittsburg Sleep Quality Index and Epworth Sleepiness Scale Value and summarized the online survey [4].

Denmark stood out from the crowd, there were no elevated suicides rates among the veterinarians [5].

Sadly, there is no survey done by any Asian Country, which might show the suicide rate of veterinarian.

Programs like Not One More Vet by Dr. Carrie Jurney and Peace Within Mindfulness by Dr. Sarah Montgomery and others have initiated to work for the upliftment and creating awareness for mental health and issues in veterinarians [6-8].

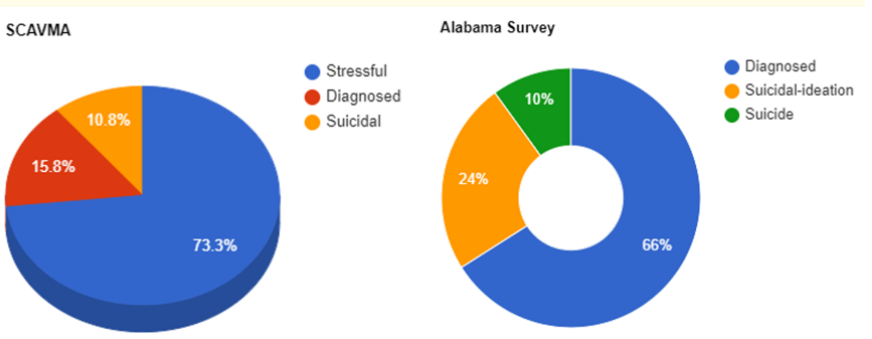

Figure 1: Graphical Representation of Veterinary Suicide - SCAVMA and Alabama State Veterinary Association's Survey.

\section{Conclusion}

Veterinarians need to priorities their work-life balance, sleep cycle, anxiety and related-issues, using various tools such as timeto-time vacation, seeking professional help in-dire need, and indulging into life-rejuvenating activities and exercises such as yoga, meditation, swimming, running, trekking etc.

Different agencies need to wake-up from their sleep and find an exact or near to it solution to eradicate or to minimize the problem of mental health issues in Veterinary students as well as Veterinary Professionals. 


\section{Acknowledgements}

Nobody has been more important to me in the pursuit of this project than the members of my family. I would like to thank my parents, siblings, whose love and guidance are with me in whatever I pursue. They are the ultimate role models.

\section{Bibliography}

1. Gregory E Skipper and Jerome B Williams. "Failure to Acknowledge High Suicide Risk among Veterinarians". Journal of Veterinary Medical Education. JVME 44.1 (2017).

2. Carlton Gyles. "Mental Health and Veterinary Suicide". Canada Veterinary Journal 55.12 (2014).

3. World Health Organization (WHO) "Mental Disorders" (2019).

4. A McKenzie., et al. "An evaluation of a veterinary-specific mental health service". Oxford Academia- Occupational Medicine 70.3 (2020).

5. Susan M Rhind and Andrew Grant. "From Studying the Rain to Studying the Umbrella: Mental Health and Well-Being of Veterinary Medical Students and Graduates". Journal of Veterinary Medical Education. JVME 44.1 (2017).

6. NPR "Veterinarians Are Killing Themselves. An Online Group Is There to Listen and Help" (2019).

7. Andrea Fournier and Katherine Goldberg. "When Working with Animals Can Hurt Your Mental Health". American Psychological Association (2019).

8. Michael T Napier., et al. "Sleep Quality and Sleepiness Among Veterinary Medical Students Over an Academic Year". Frontiers Veterinary Science 6 (2019).

Volume 3 Issue 9 September 2021

(C) All rights are reserved by Amaan Ghazi.

Citation: Amaan Ghazi. "Veterinarian and Mental Health". Acta Scientific Veterinary Sciences 3.9 (2021): 69-71. 